L. Picolli, A. Rossini, P. Malcovati, F. Maloberti, F. Borghetti, A. Baschirotto: "A Clock-Less 10bit Pipeline-Like A/D Converter for Self-Triggered Sensors"; IEEE Journal of Solid-State Circuits, Vol. 43, Issue: 2, Feb. 2008, pp. 312-320.

(C)20xx IEEE. Personal use of this material is permitted. However, permission to reprint/republish this material for advertising or promotional purposes or for creating new collective works for resale or redistribution to servers or lists, or to reuse any copyrighted component of this work in other works must be obtained from the IEEE. 


\title{
A Clock-Less 10-bit Pipeline-Like A/D Converter for Self-Triggered Sensors
}

\author{
Luca Picolli, Student Member, IEEE, Andrea Rossini, Senior Member, IEEE, Piero Malcovati, Senior Member, IEEE,
} Franco Maloberti, Fellow, IEEE, Fausto Borghetti, and Andrea Baschirotto, Senior Member, IEEE

\begin{abstract}
In this paper, a novel 10-bit A/D converter based on a pipeline-like architecture specific for low-noise, self-triggered sensors, (e.g., X-rays and $\gamma$-rays spectrometry) is presented. The main innovative feature of the proposed A/D structure is the concept that, for a sampled input signal, a pipeline ADC may behave as a combinatorial logic and may operate without any timing signal (clock). The conversion is obtained asynchronously propagating the partial conversions and the residues through the various stages. This concept is validated by means of a prototype ADC fabricated in a standard $0.35 \mu \mathrm{m}$ CMOS technology. The active area is $2.24 \mathrm{~mm}^{2}$, and it provides a conversion in $2.5 \mu$ s (i.e., it can operate with a $400 \mathrm{kS} / \mathrm{s}$ data rate) featuring an ENOB equal to 8.91 .
\end{abstract}

Index Terms-Analog-digital conversion, asynchronous logic circuits, gamma-ray spectroscopy, spectroscopy, X-ray applications.

\section{INTRODUCTION}

$\mathbf{X}$ -RAY and $\gamma$-ray spectrometry is gaining relevance in many fields, ranging from astronomy to medicine. In such spectrometers, a large array of semiconductor sensors is used to detect events, which occur randomly with Poissonian distribution. In order to build an energy spectrum, the energy of each event has to be measured with a resolution of the order of $8-10 \mathrm{~b}$. Thus, the sensors are typically connected to very-low-noise front-end circuits, which provide an output dc voltage proportional to the energy of the event (through a peak-and-hold circuit) as well as a trigger signal that informs the system that an event has occurred. Therefore, spectrometers are a typical case of self-triggered application. The integrated circuits for X-ray or $\gamma$-ray spectrometers available so far [1], [2] provide as output a static analog signal proportional to the energy of the event, while A/D conversion of this static analog signal is performed externally, typically with multichannel analyzers. The use of an external ADC introduces some noise sources due to the coupling to the analog interfacing. However, the clock signal needed for on-chip A/D conversion would introduce significant on-chip noise through the substrate that

Manuscript received December 13, 2006; revised September 24, 2007.

L. Picolli, A. Rossini, and P. Malcovati are with the Department of Electrical Engineering, University of Pavia, 27100 Pavia, Italy (e-mail: luca.picolli@ unipv.it; andrea.rossini@unipv.it; piero.malcovati@unipv.it).

F. Maloberti is with the Department of Electronical Engineering, University of Pavia, 27100 Pavia, Italy (e-mail: franco.maloberti@unipv.it).

F. Borgetti is with FBK-irst, 38050 Povo (TN), Italy (e-mail: borghetti@ fbk. edu).

A. Baschirotto is with the Department of Innovation Engineering, University of Lecce, 73100 Lecce, Italy (e-mail: andrea.baschirotto@unile.it).

Digital Object Identifier 10.1109/JSSC.2007.914249 would dramatically affect the accuracy performance of the front-end circuit. On the other hand, A/D converters, which do not require a clock signal if the input signal is already sampled, such as full flash A/D converters, do not achieve a sufficient accuracy for spectrometry. Therefore, a fast and accurate A/D converter capable of operating without a clock signal would be extremely useful to achieve high-performance fully integrated X-ray or $\gamma$-ray spectrometers. These devices would be beneficial especially for astronomical applications on satellites, where size and weight have to be minimized.

The innovative clock-less A/D converter presented in this paper allows one to solve the above bottleneck in the design of self-triggered sensor applications. The proposed A/D architecture is based on the pipeline structure, which can achieve a good accuracy (up to 10 bits). The typical pipeline architecture has been properly modified in order to operate without the clock signal. The proposed structure requires that the input signal has to be sampled and a trigger signal is provided to start the conversion. These requirements are fully satisfied by the self-triggered sensor applications, among which Fig. 1 reports a possible block diagram.

The main innovative feature of the proposed structure is that this pipeline ADC behaves as combinatory logic. In the data converters so far, the task of a clock signal is to give proper timing to the sequence of operations required to achieve the conversion from the analog to the digital domain or vice versa. If it is assumed that each operation is completed within a certain time slot, then the clock can be removed, thus obtaining the proposed structure. The proposed novel concept requires an improved block design in order to guarantee timing operation as it will be discussed in the following.

This paper is organized as follows. After this introduction, Section II presents the clock-less concept and the relative modification of the ADC building blocks. Section III gives details about the circuit design of the ADC. Section IV describes the realization of a silicon prototype that demonstrates the validity of the proposal. Layout issues and experimental results are reported. Section V concludes the paper.

\section{Clock-Less PiPeline-Like ARChitecture}

The architecture of the proposed clock-less pipeline-like A/D converter is shown in Fig. 2. This structure appears as a standard pipeline ADC. The architecture consists of nine conversion stages, an array of flip-flops, and a ripple-carry adder, which provides the digital correction to reduce threshold voltage variation sensitivity. Each of the first eight stages provides 1.5 bits of partial conversion, while the last one is a 2-bit flash ADC. The output bits of the nine stages are sampled by a register of flip- 


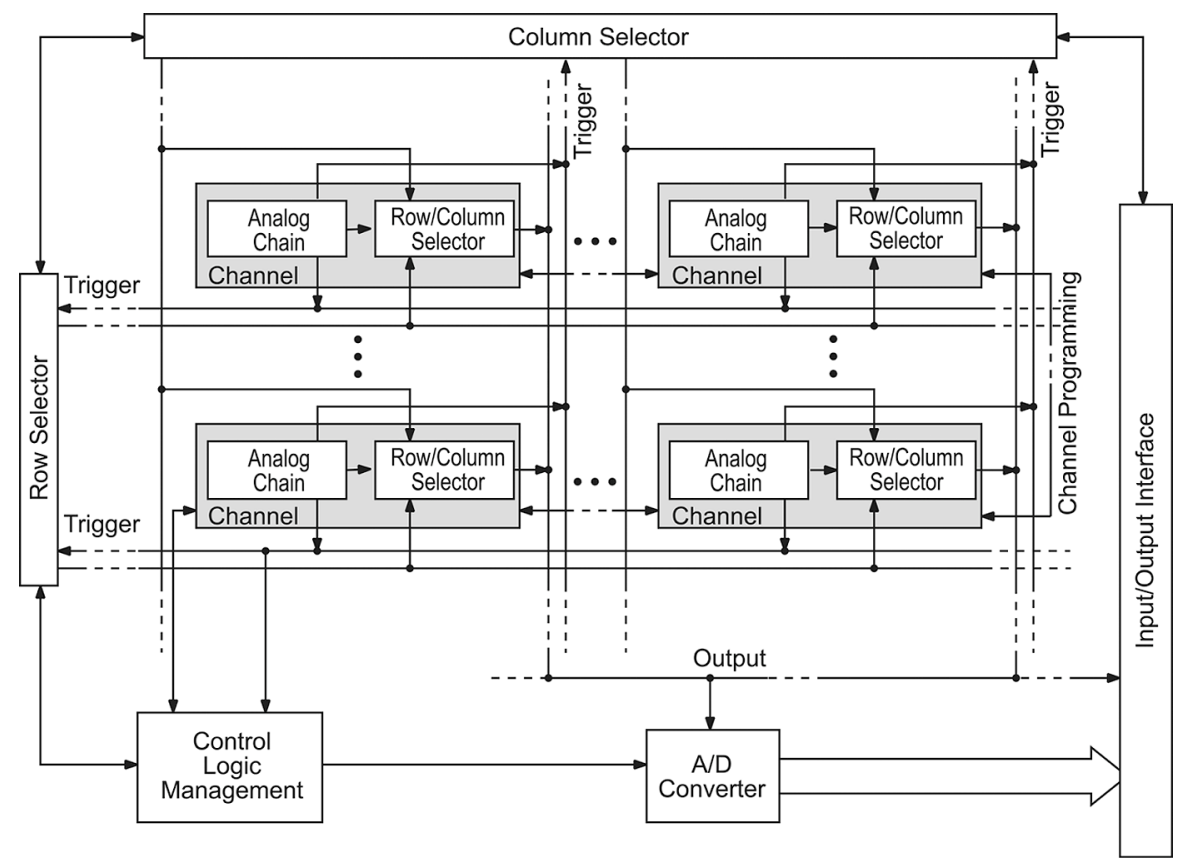

Fig. 1. Typical application of the proposed A/D converter.

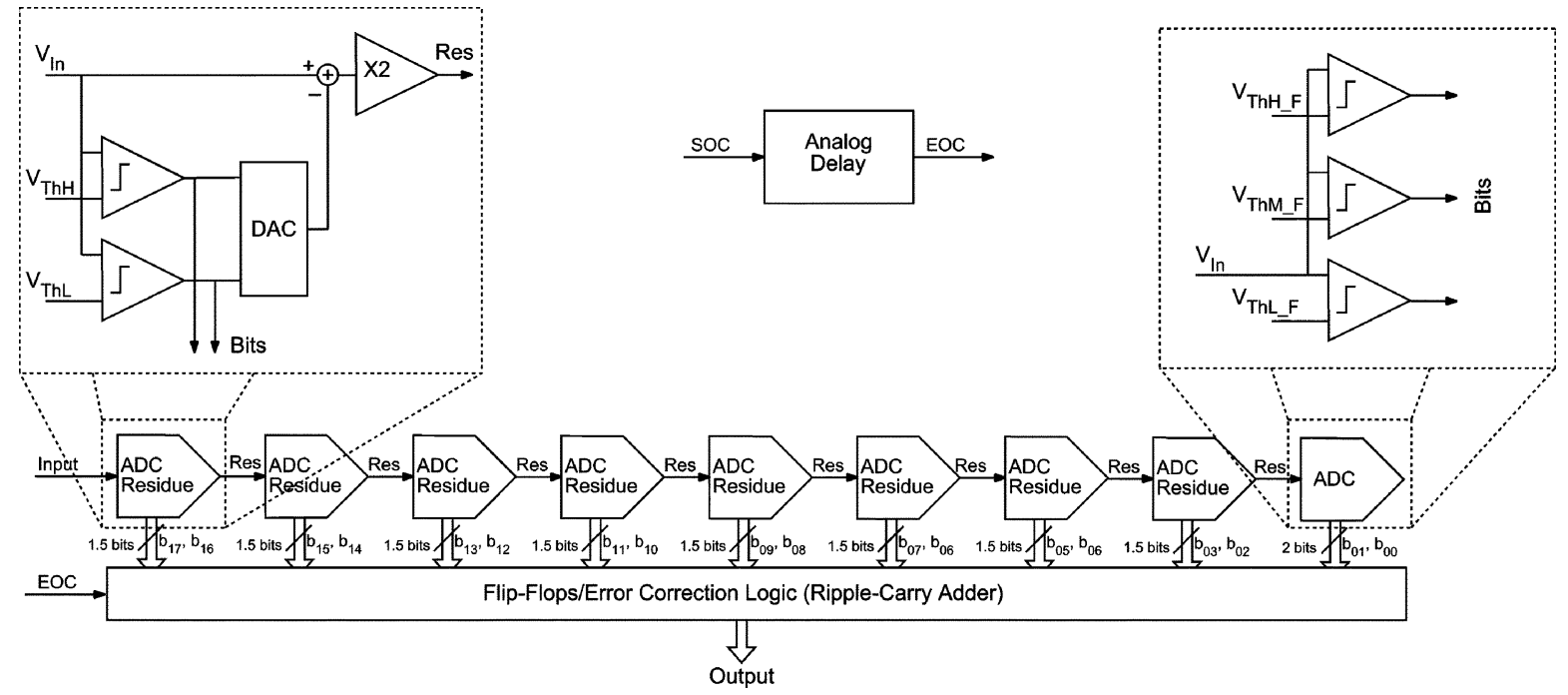

Fig. 2. Block diagram of the A/D converter.

flops controlled by the end-of-conversion (EoC) signal, which is a delayed version of the start-of-conversion (SoC) signal, and added with a ripple-carry adder (RCA) in order to achieve the final ten output bits with digital error correction [3]. The schematic diagram of one of the first eight stages as well as that of the last stage are shown in the insets of Fig. 2. Each 1.5 bit conversion stage consists of a flash ADC with a resolution of 1.5 bits, a DAC, an adder, and a gain stage with a gain equal to 2 .

The key requirement of the circuit design is to ensure that the overall ADC data conversion ends within the scheduled time slot. The most critical point to achieve this target is the calculation of the residual error at each 1.5 bit stage for the following stage, which is operated using the results of the previous stage (with the eventual error propagation). The overall data conver- sion time slot is obtained adding the residual calculation time of each of the nine stages of conversion.

The main difference with respect to a standard pipeline ADC is within the comparator and ADC functionality and, as a consequence, in the circuit design.

The general functionality of a comparator in a pipeline ADC is two-fold:

1) to take a decision within the available time slot;

2) to maintain the output signal for the needed time for the following stage operation.

These two features in a standard clocked comparator are realized by means of the following solutions.

1) The comparator uses a structure composed by a preamplifier and a latch. The latch operates on an amplified signal 


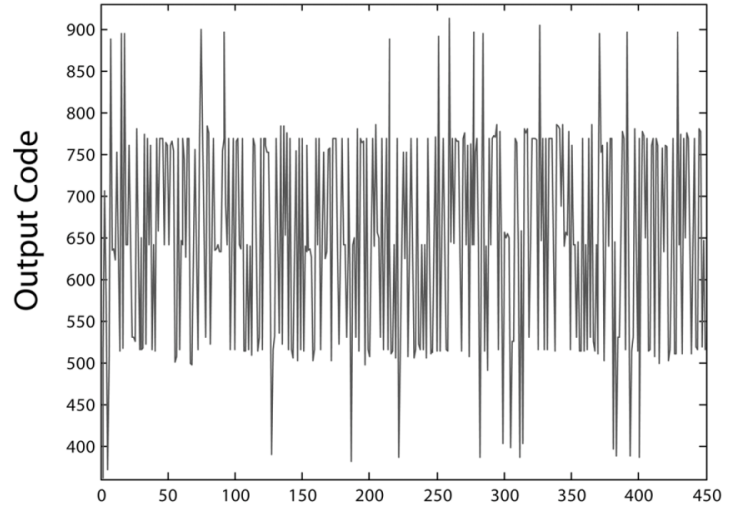

a)

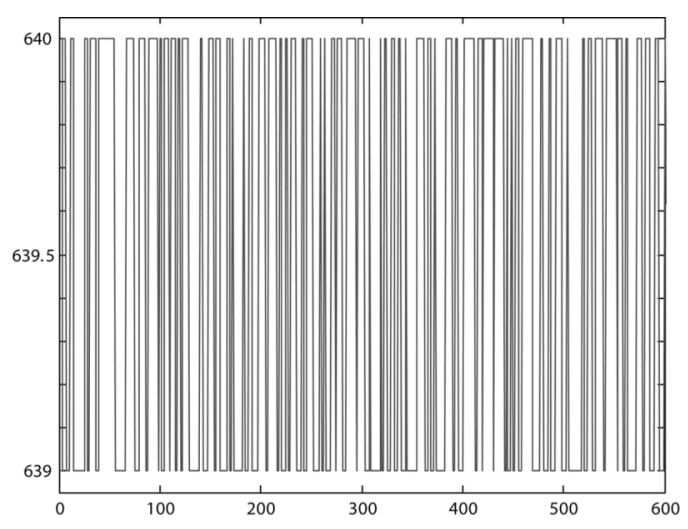

b)

Time $[\mu \mathrm{s}]$

Fig. 3. Output code applying a constant input with a 1-LSB amplitude noise added (a) without and (b) with hysteresis.

(the preamplifier output) and embeds a positive feedback to increase the gain and speed-up the decision.

2) The clock signal commutes between different states of a register that reads the latch output signal during one clock phase and properly maintain this signal during the other clock phase.

The operations of these two blocks are strictly correlated with the use of the clock signal that drives them. In the proposed structure, the clock is not available. As a consequence, the two above features have to be realized with an alternative approach. In this paper, a possible solution is proposed for the two points, as follows.

1) The comparator structure uses only an open-loop amplifier stage, whose output will be used as a digital signal. Such an amplifier has to feature very large gain (much larger than the preamplifier of a clocked comparator) to guarantee a decision even for the minimum signal within the available time slot and to prevent having metastability of the comparator.

2) Since the comparator input signal could, in principle, change during operation (due to noise or disturbances), due to the comparator open-loop operation, the comparator output signal could change with a consequent error in the following stage operation. A proper hysteresis has then been implemented in the comparator. Thus, once a decision is taken, the comparator does not change its output signal. The exact value of the hysteresis will be analyzed later on.

These two arrangements guarantee the functionality of the overall pipeline-like clock-less ADC. The validity of the proposal is demonstrated in Fig. 3 that shows the output code while applying a constant input with a 1-LSB amplitude noise added, with and without hysteresis (simulations in Simulink). No effect of the applied noise is observed in the ADC using the hysteresis. On the other hand, without hysteresis, the output code shows a ripple much larger than $1 \mathrm{LSB}$, reducing ADC performance.

As a result of the above considerations and proposals, the 1.5-bit ADC within each pipeline stage is realized with two open-loop comparators with hysteresis [4], which compare the input signal with two thresholds.
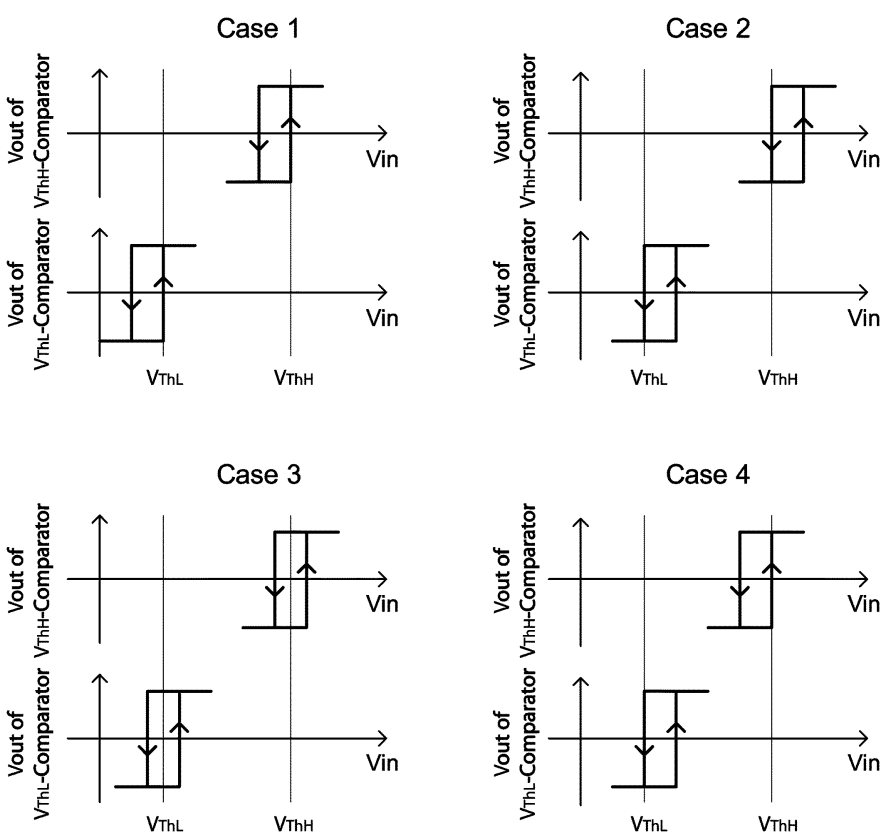

Fig. 4. Four different types of hysteresis simulated.

Notice that the digital correction allows the A/D converter to operate properly even in the presence of a large threshold error. This is greatly exploited in this solution, since hysteresis can be considered as a threshold error. The form and the value of the hysteresis have been chosen after widely simulating in Simulink an ideal model of the A/D converter with different possible solutions of hysteresis in the two 1.5-bit ADC comparators. In this analysis, the actual full-scale signal of the ADC $\left(2 \cdot V_{\mathrm{REF}}=1.2 V_{\mathrm{pp}}\right)$ is used. In this case, the nominal values of the threshold voltages of the two comparators are placed at $V_{\mathrm{REF}} / 4=+150 \mathrm{mV}$ and $-V_{\mathrm{REF}} / 4=-150 \mathrm{mV}$, respectively.

Four different forms of hysteresis have been simulated, the schemes of which are shown Fig. 4, and are:

- aligned to the bottom on both the comparators of the 1.5-bit ADC (case 1);

- aligned to the top on both the comparators of the 1.5-bit ADC (case 2); 


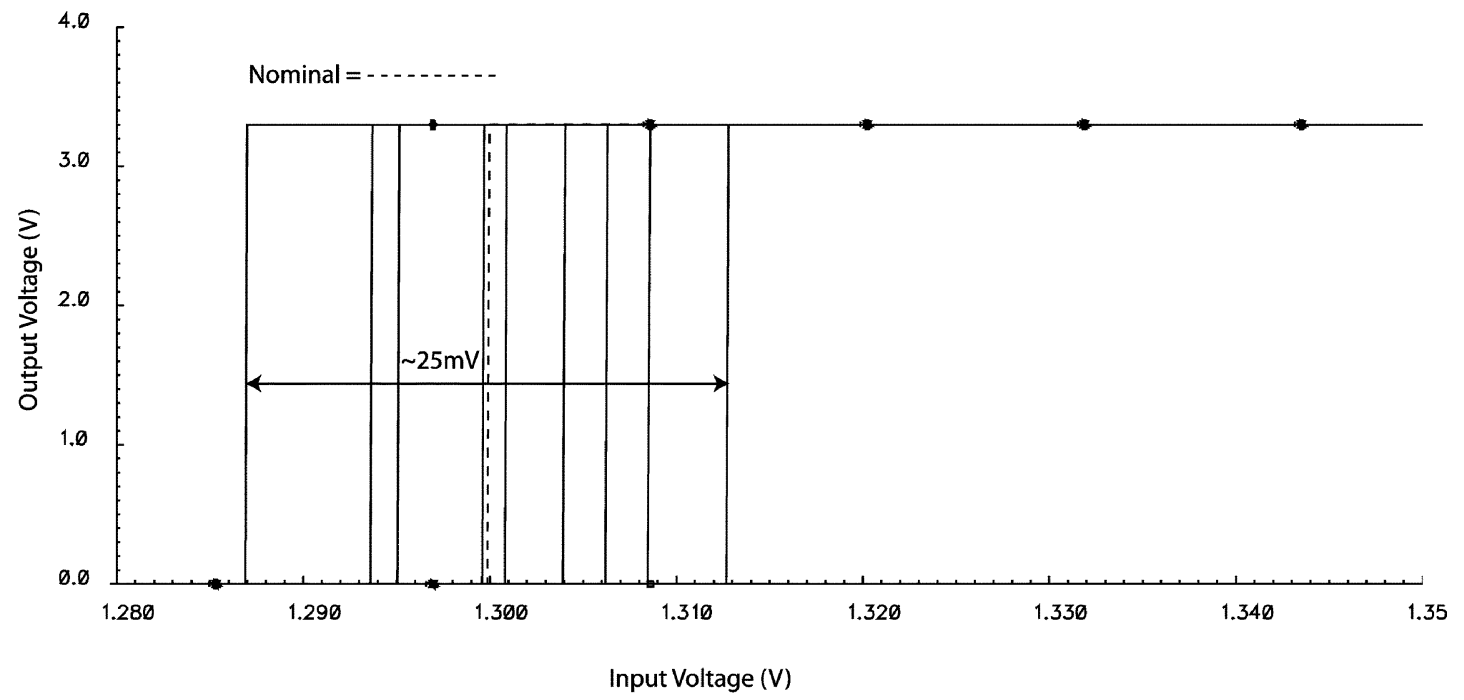

Fig. 5. Comparator response in the process corners.

TABLE I

MAXIMUM VALUE OF THE HySTERESIS

\begin{tabular}{|l|c|c|c|c|}
\hline & \multicolumn{4}{|c|}{ Simulation results } \\
\cline { 2 - 5 } & Case1 & Case2 & Case3 & Case4 \\
\hline Maximum value of hysteresis & $150 \mathrm{mV}$ & $140 \mathrm{mV}$ & $150 \mathrm{mV}$ & $140 \mathrm{mV}$ \\
\hline
\end{tabular}

- aligned centred on both the comparators of the 1.5-bit ADC (case 3);

- aligned to the bottom on the $V_{\mathrm{ThH}}$-comparator and aligned to the top on the $V_{\mathrm{ThL}}$-comparator (case 4).

Table I shows the maximum value of the hysteresis allowed in the four cases to ensure either DNL or INL to be within $-0.5 \mathrm{LSB}$ and $0.5 \mathrm{LSB}$.

As a consequence, the form of the hysteresis has been selected considering other implementation issues. In this design, a centered hysteresis (case 3) around the threshold voltage level has been used because of its layout symmetry that gives the design robustness.

On the other hand, the amount of the hysteresis has been selected with functionality considerations. In the above analysis, for all of the cases, the structure performance becomes critical for a hysteresis larger than $150 \mathrm{mV}$. These values corresponds to the considerations that the hysteresis has to be lower than the reference voltage of the comparators, otherwise the digital correction is no longer effective. In fact, since the digital correction technique corrects threshold errors up to $V_{\mathrm{REF}} / 4(=150 \mathrm{mV}$ for $V_{\mathrm{REF}}=0.6 \mathrm{~V}$ ) [3], all of the above cases respect the 1.5-bit per stage theory of an $\mathrm{A} / \mathrm{D}$ pipeline converter. In this design, the amplitude of the hysteresis is set to $100 \mathrm{mV}$ as a tradeoff between large robustness for the clock-less operation (achieved with a large hysteresis) and good digital correction effectiveness (achieved with small hysteresis).

Simulations in the worst case were made for the comparator to be sure that the value of the hysteresis does not exceed the maximum tolerable value of $V_{\mathrm{REF}} / 4\left(V_{\mathrm{REF}}=0.6 \mathrm{~V}\right)$. Fig. 5 shows the falling decision voltage of the $V_{\text {ThL }}$-comparator, ideally equal to $1.3 \mathrm{~V}$, in several process corners. It can be noticed that the comparator decision is almost the same in every process corner. The variation of the decision voltage is around $25 \mathrm{mV}$, which stays in the limits of the digital correction. Thus, the nominal value of $100 \mathrm{mV}$ guarantees that, for any worst case (e.g., aging, temperature, or technology), the hysteresis is lower than that of $V_{\mathrm{REF}} / 4$.

The only digital signal in the proposed clock-less pipelinelike $\mathrm{ADC}$ is the $\mathrm{SoC}$ that is generated by the experiment trigger. A second control signal is the EoC, which is obtained by delaying the SoC signal through an analog block, consists of an $R C$ network followed by a comparator equal to those used in the main ADC. The value of delay is equal to $T_{\text {sample }}$ and has been chosen in order to match the delay of the pipeline chain. This signal is used to strobe the digital output of each stage and to control the digital correction section.

\section{Clock-Less Pipeline-Like CiRCUIT DesigN}

Here, a detailed description of the most important circuit solutions is given.

The schematic diagram of the comparator is shown in Fig. 6. The key point regards the hysteresis amount, as previously analyzed, that has been implemented at the transistor level. The proposed comparator operates as follows where the value of the hysteresis is proportional to the ratios $\beta_{10} / \beta_{3}$ and $\beta_{11} / \beta_{4}$.

Assume that initially the input voltage $V_{1 n}$ is much lower than the threshold voltage of the comparator. In this case, all of the current flows through MN1 and MP3; MP11 and MP4 are off, and consequently the output voltage is low. MP10 is on also, but no current is flowing in it. Initially, when the input voltage increases, nothing happens until $V_{I n} \cong V_{\text {Th }}$. At this point, some current starts to flow into MN2 from MP10 and $I_{N M 1}$ starts to decrease, so the following relations can be written:

$$
\begin{aligned}
I_{M N 2} & =I_{M P 10}=\alpha_{r} I_{M P 3} \\
I_{M N 1}+I_{M N 2} & =I_{M N 5}=I_{\mathrm{Bias}} \\
I_{M N 1}+\alpha_{r} I_{M P 3} & =I_{\mathrm{Bias}} \\
I_{M N 2} & =\alpha_{r} I_{M P 3} \propto\left(V_{\mathrm{In}}-V_{\mathrm{Th}}\right)^{2}
\end{aligned}
$$




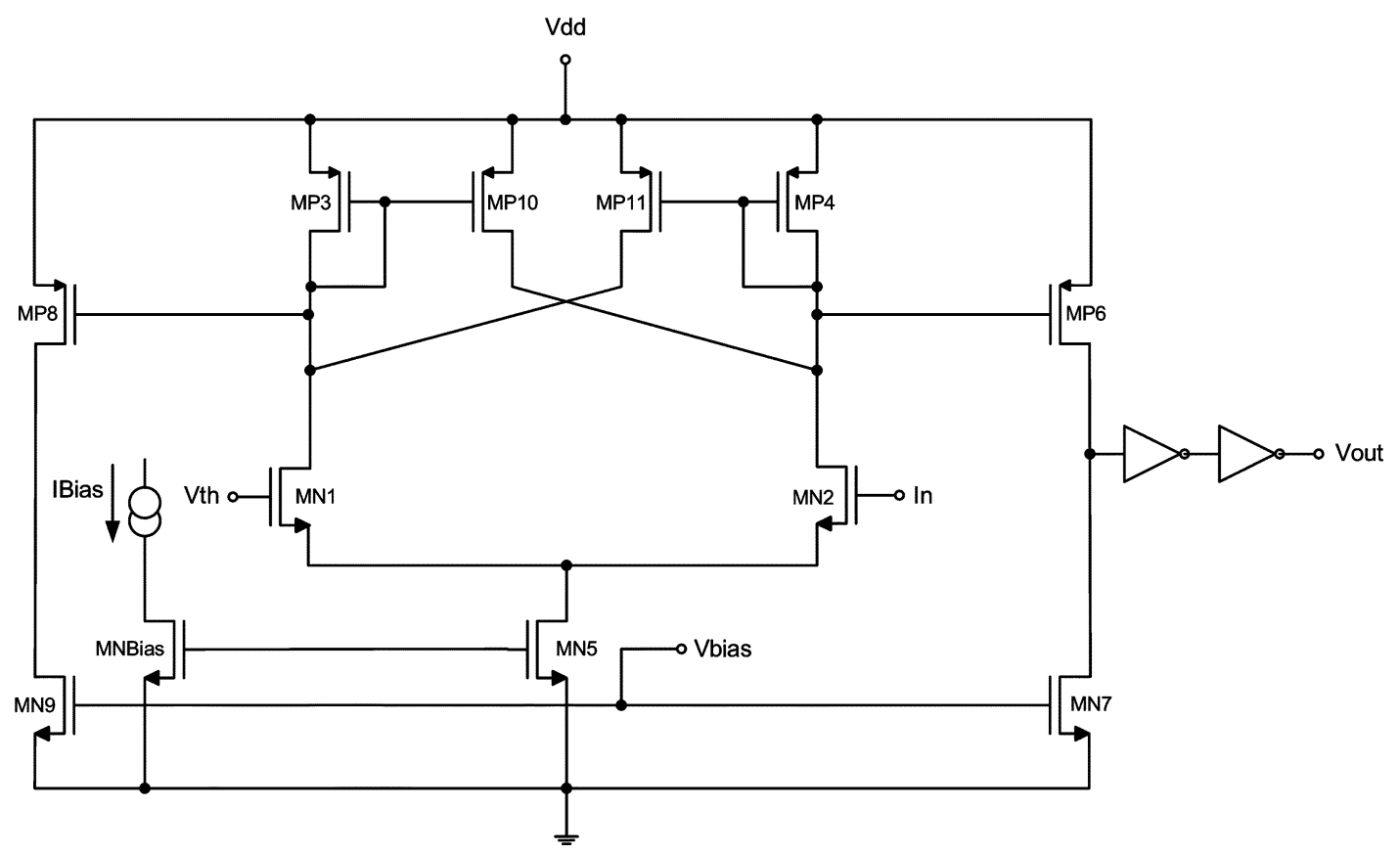

Fig. 6. Schematic circuit of the comparator.

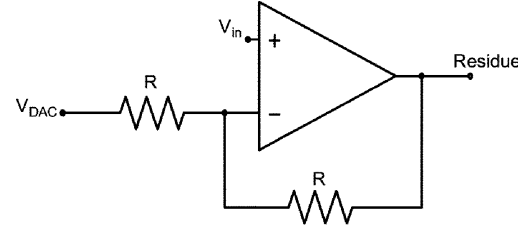

(a)

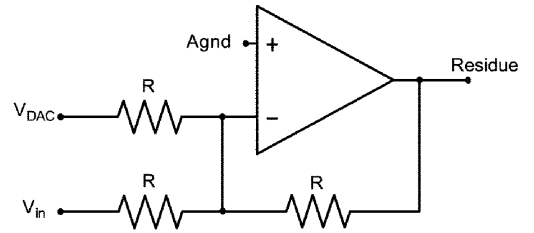

(b)

Fig. 7. The adder and gain stage. (a) Noninverting. (b) Inverting.

where $\alpha_{r}$ is the ratio $\beta_{10} / \beta_{3}$, and subscript $r$ stands for raising. If the input voltage is increased further on, MN2 demands for more current that can come only from MP10 that mirrors $I_{M P 3}$ with the ratio $\alpha_{r}$ while $I_{M P 3}$ is decreasing. At a certain point, $\operatorname{MP10}\left(\alpha_{r} I_{M P 3}\right)$ cannot validate (2), because $I_{M P 3}=I_{M N 1}$ is too low, and consequently MP4 goes on, providing the current MN2 is asking for [see (4)]. Therefore, the output voltage of the comparator becomes high as follows:

$$
I_{M N 1}+I_{M P 4}=I_{\text {Bias }}
$$

The last value of $V_{\text {In }}$ that verifies (2) is the raising-decision threshold, and the difference $V_{\mathrm{In}}-V_{\mathrm{Th}}$ is the value of the raising-hysteresis that is proportional to $\alpha_{r}$. Thus, its value can be changed by the $\alpha_{r}$ parameter: the higher $\alpha_{r}$ is, the longer (2) is verified and higher the raising-hysteresis that validates (3) is. It is important to point out that (1)-(3) are true only when $V_{\text {In }} \cong V_{\text {Th }}$ and (4) is true only when the output voltage is high.

The same operations happen symmetrically in the falling decision: the falling hysteresis is determined by $\alpha_{f}$ that is the ratio $\beta_{11} / \beta_{4}$, and subscript $f$ stands for falling. MP11, MP4, and MN2 are on while MP3, MP10, and MN1 are off.

The transistor sizes of the comparator are shown in Table II. The gain of this stage is about $30 \mathrm{~dB}$. This is not sufficient to
TABLE II

DIMENSIONS OF THE COMPARATOR'S MOSFET

\begin{tabular}{|c|c|}
\hline Transistors & W/L $(\mu \mathrm{m} / \mu \mathrm{m})$ \\
\hline MN1,MN2,MP3,MP4 & $35 / 0.35$ \\
\hline MN4, MN7, MNBias, MP6, MP8 & $40 / 0.35$ \\
\hline MN5 & $50 / 0.35$ \\
\hline MP10, MP11 & $100 / 0.35$ \\
\hline
\end{tabular}

guarantee a decision within the available time slot for the minimum input signal. Two digital inverters have then been added at the comparator output to guarantee the required gain.

The MDAC subtracts the voltage generated by the DAC from the input signal, generating the residual voltage, which is amplified by two, and represents the input signal of the next stage. Also, in this stage, the use of a nonclocked structure results in novel implementation solutions. The adder and the gain stage can be realized with an operational amplifier in closed-loop configuration with two possible solutions, as shown in Fig. 7. The designed solution is the noninverting one, as will be discussed in Section IV. The two resistors used in the feedback path are of the same value and equal to $100 \mathrm{k} \Omega$. This value is sufficiently large to avoid a significant increase of the operational amplifier power consumption and small enough to require reasonable area 


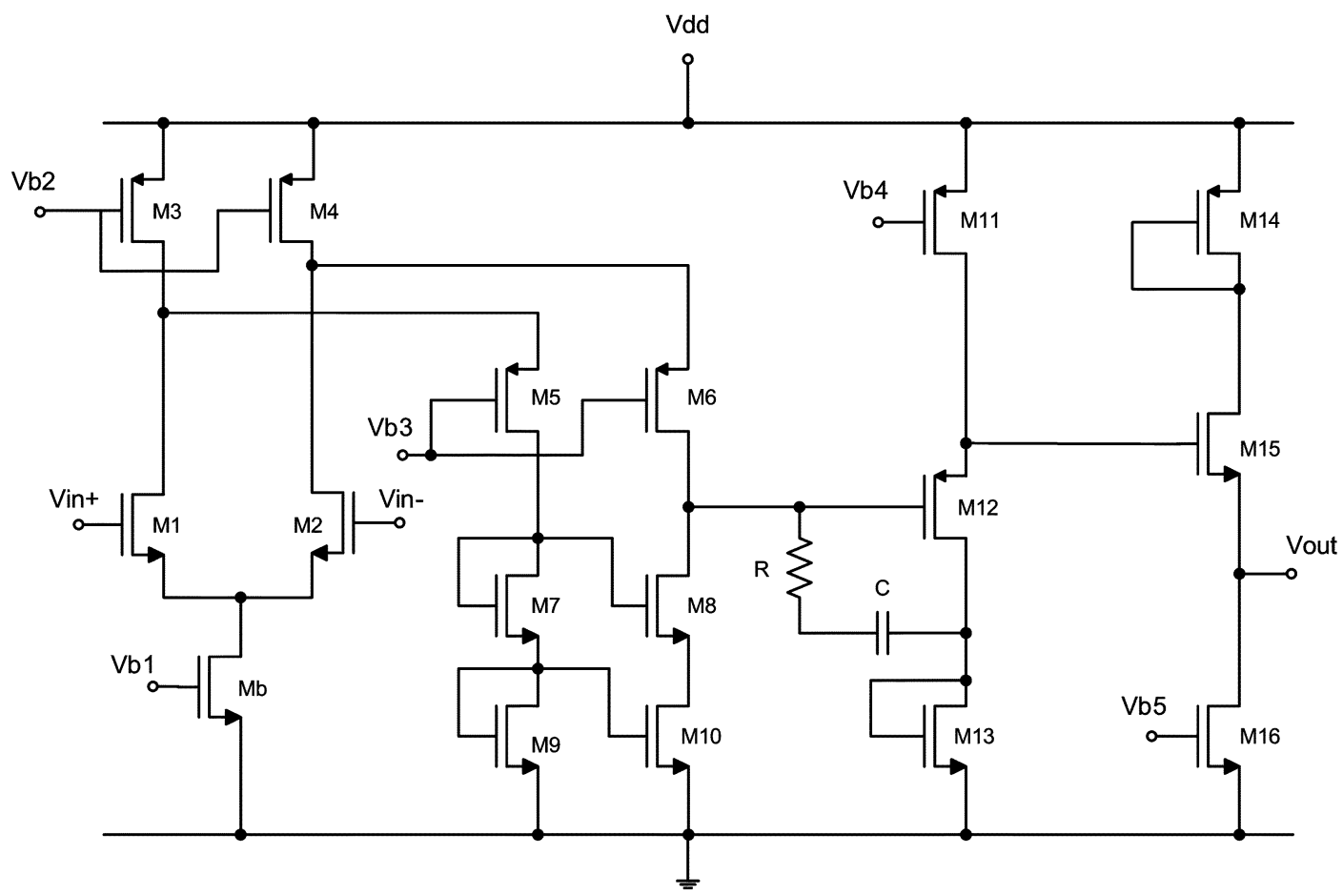

Fig. 8. Structure of the operational amplifier.

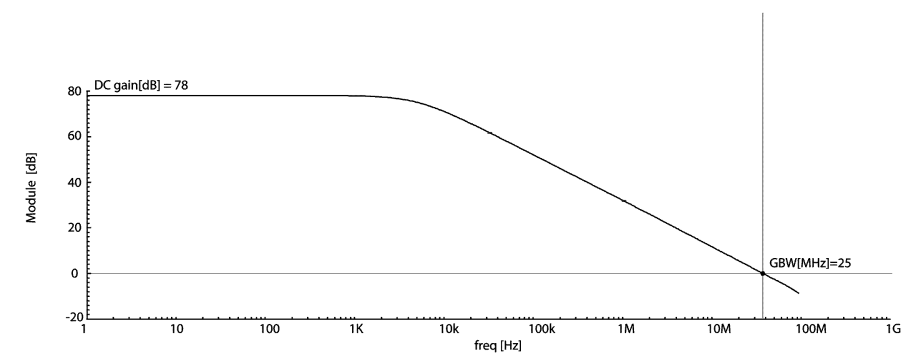

Fig. 9. Frequency response of the operational amplifier.

occupancy. The use of this structure results in the additional requirement for the operational amplifier to feature the same input and output common-mode voltage. As a consequence, its input range has to be large.

For this reason, a single-ended folded-cascode structure with a low-impedance output stage shown in Fig. 8 is used. In fact, it guarantees sufficiently large output swing, large dc gain, and the possibility of having the same input and output commonmode voltage. The output branches, made by the transistors M11, M12, M13, M14, M15, and M16 provide a level shifter to ensure the same common mode as the input's and ensure a low-impedance output as stated earlier. Therefore, the dc gain of the operational amplifier is preserved for degradation due to a finite value of the feedback resistance.

The frequency response of the operational amplifier of Fig. 8 is shown in Fig. 9, assuming a $2 \mathrm{pF}$ load that corresponds to the worst case load when the MDAC is operating in the actual ADC structure.

The last conversion stage of the pipeline structure is a 2-bit flash ADC, which consists of three open-loop comparators, which divide the input range into four intervals, providing two

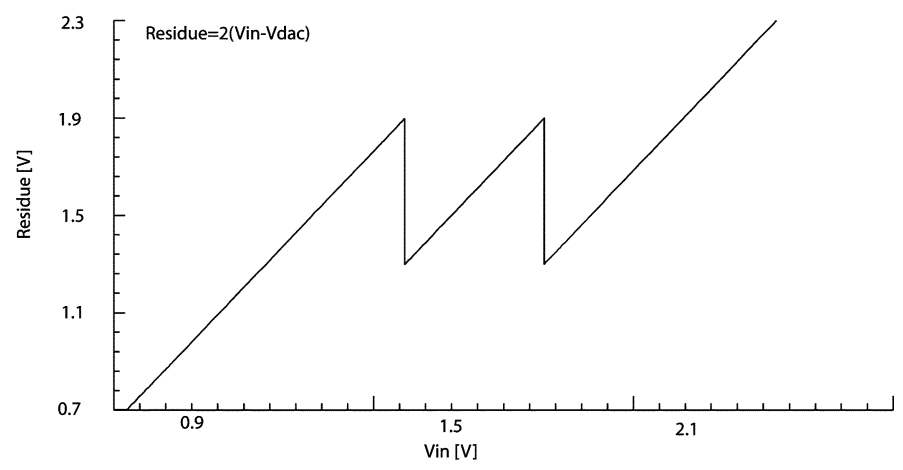

Fig. 10. 1.5 bit stage transfer function at the simulation level.

bits of conversion. The 18 bits provided by the nine conversion stages are sampled by an array of flip-flops driven by the EoC signal.

The transfer function of the 1.5 bit stage is shown in Fig. 10. The plot is obtained from a Cadence simulation.

\section{A. Conversion Speed and Architecture Limits}

The conversion speed limit depends on the speed response of the comparators and of the amplifier in the MDAC, as discussed in the following.

Due to the multistage structure of the comparator, its speed is very high and it is, in any case, much higher than the MDAC speed. The hysteresis presence also guarantees response robustness to noise, as shown in Fig. 3.

The conversion speed limit is then given by the amplifier in the MDAC. In this stage, the operational amplifier is operating in a closed loop configuration, which fixes the speed of response. The particular operational amplifier configuration (the noninverting configuration) is preferred to the inverting one [shown 


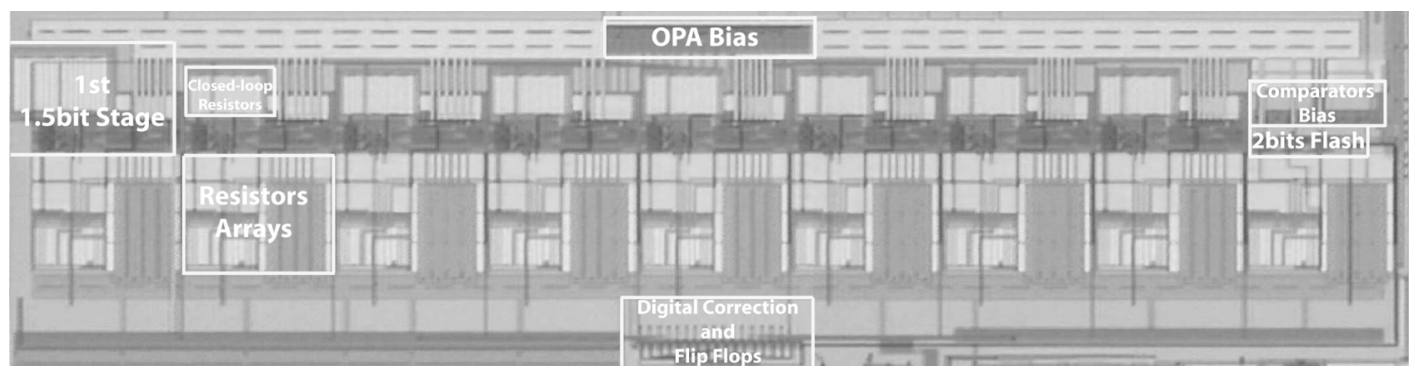

Fig. 11. Microphotograph of the chip.

in Fig. 7(b)], since it achieves a higher speed. This higher speed (due to a larger feedback factor) is obtained at the cost of the higher operational amplifier input signal swing. The feedback factor is $1 / 2$, and this gives a closed-loop bandwidth $(12.5 \mathrm{MHz})$ equal to $1 / 2$ of the operational amplifier unity gain bandwidth (about $25 \mathrm{MHz}$ ). The $9.5 \mathrm{MHz}$ pole for a 9 bit settling accuracy leads to a response time of $0.1 \mu \mathrm{s}$. The cascade of nine such stages then leads to a $0.9 \mu$ s delay, which is consistent with the overall conversion time verified in the experimental results.

It useful to point out that the proposed structure could have a potential issue of error propagation due to the fact that the comparator could decide far before the input signal is fully settled (e.g., because of the ripple of the signal during its settling). This could happen because, as mentioned in Section II, the comparator structure is continuous-time and hence it can switch at any time. From circuit simulations in Cadence environment that take into consideration this aspect (i.e., the ripple of the signal during its settling), this error does not affect the performance of the A/D converter, thus leading in simulation to an ENOB of 9.7 bits.

The conversion process with $N$ cascaded stages results in a limit of the sampling frequency that is $N$ times lower than a clocked pipeline A/D converter with the same settling time per stage.

On the other hand the proposed A/D converter, although it features a competitive power consumption in its application field (i.e., event-triggered application), burns most of the power just to maintain the residue until the conversion process is finished, while a clocked pipeline $\mathrm{A} / \mathrm{D}$ converter, with the same frequency and resolution, neglecting the latency (which is anyway important in event-triggered applications), would consume, in first approximation, $N$ time less than the clockless solution.

\section{EXPERIMENTAL RESULTS}

The proposed clock-less pipeline-like ADC concept has been demonstrated by the realization of a prototype in a standard $0.35 \mu \mathrm{m}$ CMOS technology. Fig. 11 shows the microphotograph of the active area whose size is $2.24 \mathrm{~mm}^{2}$. The eight 1.5-bit stages, the last 2 bit flash ADC, the feedback resistor arrays, the operational amplifier bias circuit, the comparator bias circuit, the digital part, and the resistor arrays for the reference voltages can be observed. A particular issue of this realization is that the input signal of the target application, X-ray and $\gamma$-ray spectrometry, is single-ended. For this reason, the full ADC topology uses single-ended structures, and this gives additional worth to the achieved $\mathrm{ENOB} \cong 9$ bit.

\section{A. Layout Issues}

In the layout design, the flash ADC is much smaller than the 1.5-bit stages because it does not include the operational amplifier and the feedback resistors. Particular attention was devoted to the design of the resistors used in the closed-loop amplifier because they determine the accuracy of the generated residue, and then any inaccuracy is reflected in the overall ADC performance. To achieve this target, each resistor consists of eight $12.5 \mathrm{k} \Omega$ stripes for a total value of $100 \mathrm{k} \Omega$ with dummy resistors around to minimize the border effects [5]. High-resistive polysilicon was used to realize the resistors because it features the lowest mismatch factor among the materials available in the used CMOS technology. The mismatch between two equal resistors can be written as

$$
\sigma\left(\frac{\Delta R}{R}\right)=\frac{A_{R}}{\sqrt{W L}}
$$

where $W$ is the width of the resistor, $L$ is the length, and $A_{R}$ is the mismatch parameter (for the high-resistive polyslicon used, $\left.A_{R}=7 \mu \mathrm{m}\right)$. To calculate the maximum allowed mismatch between the two resistors of the first stage of the pipeline structure, which is obviously the most critical since it has to guarantee 9 bit, the following relation was used for a full scale $\left(=2 V_{\mathrm{REF}}\right)$ of $1.2 \mathrm{~V}$ :

$$
\text { Mismatch }_{\max }=\frac{1.2}{2^{9}}=0.00243 .
$$

As a consequence, a maximum mismatch of $2.4 \%$ can be tolerated. The width and the length of the resistors are then determined by using the following relation:

$$
\sigma\left(\frac{\Delta R}{R}\right)=\sigma\left(\frac{8 \Delta r}{8 r}\right)=\sigma\left(\frac{\Delta r}{r}\right)
$$

where $r$ represents one of the eight $12.5 \mathrm{k} \Omega$ stripes of each resistor. Choosing $13 \mu \mathrm{m}$ as width and $130 \mu \mathrm{m}$ as length (leading exactly to $12.5 \mathrm{k} \Omega$ ), the obtained value of $\sigma$ is

$$
\sigma\left(\frac{\Delta R}{R}\right)=\frac{7}{\sqrt{13 \cdot 130}}=0.17
$$

Therefore, the mismatch on the ratio between the two resistors $\mathrm{R}$ in Fig. 7, which determines the gain $G$ of the residue amplifier, is given by

$$
\begin{aligned}
\sigma\left(\frac{\Delta G}{G}\right) & =\sqrt{\sigma\left(\frac{\Delta R}{R}\right)+\sigma\left(\frac{\Delta R}{R}\right)} \\
& =\sqrt{2} \sigma\left(\frac{\Delta R}{R}\right)=\sqrt{2} \cdot 0.17=0.24
\end{aligned}
$$




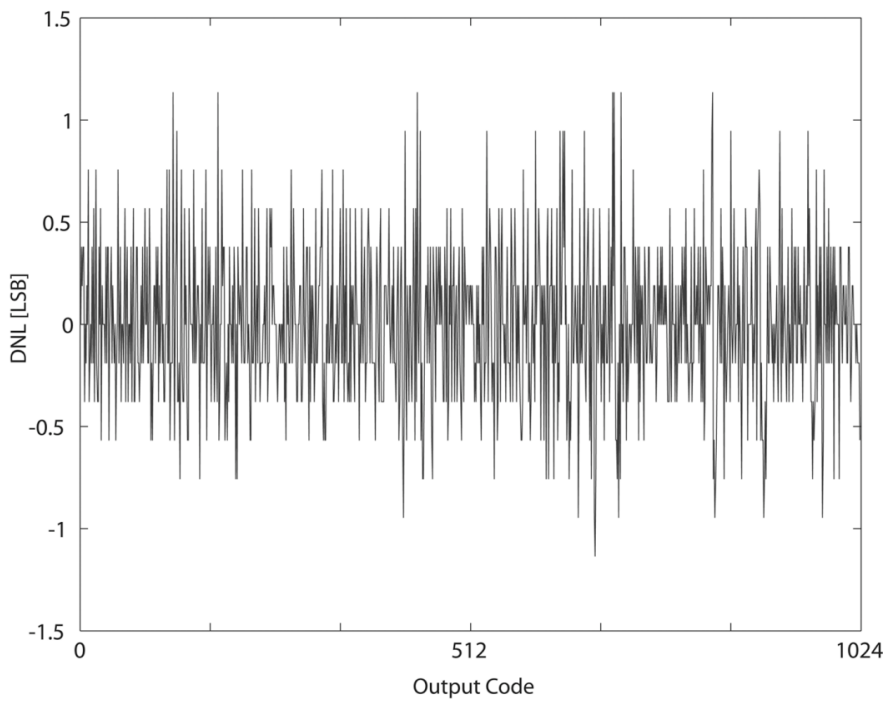

Fig. 12. DNL.

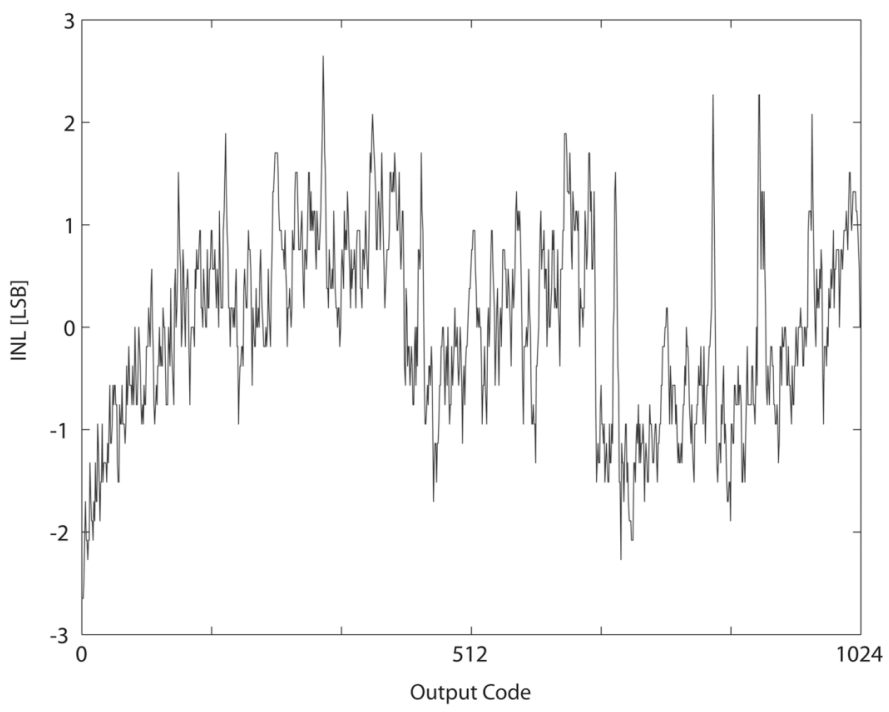

Fig. 13. INL.

which is lower than the maximum allowable value.

\section{B. Measured Performance}

The proposed A/D converter provides a conversion within a $2.5 \mu$ s time-slot after the $\mathrm{SoC}$ signal. This allows a possible data rate of $400 \mathrm{kS} / \mathrm{s}$.

To measure either static (INL and DNL) or dynamic (SNR and ENOB) performance parameters of the A/D converter, an external sample and hold was used to match the configuration of the final spectrometry application. Figs. 12 and 13 show the static performance of the converter: the DNL is within -1.2 and 1.2 LSB while the INL is within -2.5 and 2.5 LSB. This satisfies the application requirement of 8 bits.

Fig. 14 shows the dynamic performance. A typical FFT spectrum measured with an $80 \mathrm{kHz}$ input sine wave at $400 \mathrm{kS} / \mathrm{s}$ is

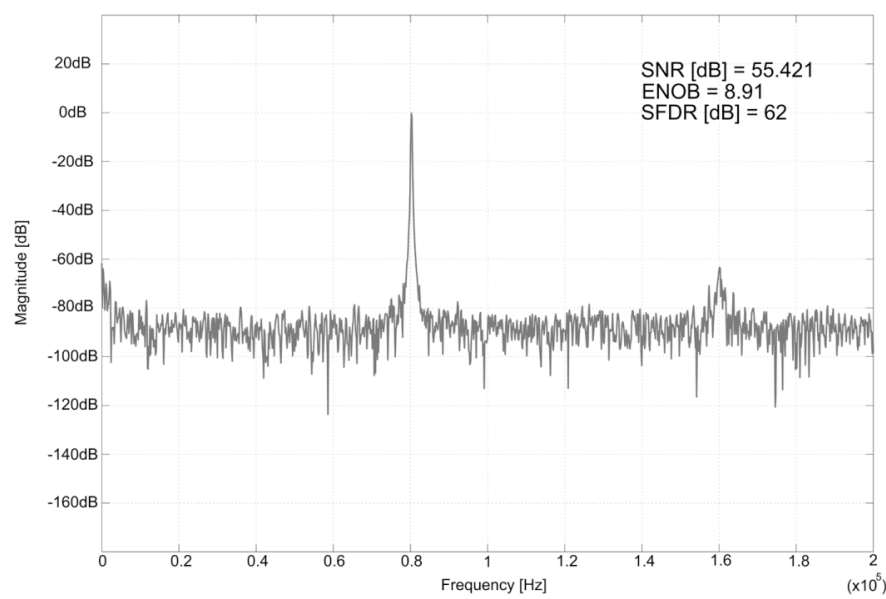

Fig. 14. Output spectrum for a $80 \mathrm{kHz}$ full-scale input signal.

reported. Notice that operating with a ratio $f_{\text {in }} / F_{\mathrm{s}}=1 / 5$ corresponds to having large steps between consecutive samples, and this could be critical for a clock-less ADC. In addition, an external sample-and-hold is not perfectly suitable for this timing-less converter. However, in this case, an $\mathrm{SNR}=55.4 \mathrm{~dB}$ (i.e., an ENOB $=8.91$ bit) is achieved, demonstrating the validity of the proposed ADC concept and implementation.

The power consumption is $14 \mathrm{~mW}$ from a $2.3 \mathrm{~V}$ power supply. Nonetheless, this power amount is significantly large with respect to other 10 bit $400 \mathrm{kS} / \mathrm{s} \mathrm{ADC}$, and this value is competitive for the considered application in comparison with alternative solutions that should have larger power consumption for exhibiting the same performance of environment clarity achieved here with the clock-less concept.

\section{CONCLUSION}

In this paper, a novel $\mathrm{A} / \mathrm{D}$ converter concept for low-noise, self-triggered applications is proposed. The main innovative feature of this $\mathrm{A} / \mathrm{D}$ converter is that, assuming that a sampled input signal is provided, it operates without the clock signal, which is usually a source of severe disturbances in low-noise circuits. The A/D converter in a standard $0.35 \mu \mathrm{m}$ CMOS technology with an active area of $2.24 \mathrm{~mm}^{2}$ provides a conversion every $2.5 \mu \mathrm{s}(400 \mathrm{kS} / \mathrm{s})$ and consumes $14 \mathrm{~mW}$ from a $2.3 \mathrm{~V}$ power supply.

\section{REFERENCES}

[1] S. Baba, K. Ohmori, Y. Mito, T. Tanoue, S. Yano, K. Tokumori, F. Toyofuku, and S. Kanda, "Recent development of radiation measurement instrument for industrial and medical applications," Nuclear Instruments and Methods in Physics Research Section A: Accelerators, Spectrometers, Detectors and Associated Equipment, vol. 458, no. 1, pp. 262-268, 2001

[2] M. Prydderch and P. Seller, "A 16 channel analogue sparse readout IC for integral," in Proc. IEEE Nucl. Sci. Symp. Med. Imag. Conf., 1994, vol. 1, pp. 65-68.

[3] R. van de Plassche, Integrated Analog to Digital and Digital to Analog Converters, 2nd ed. Boston, MA: Kluwer, 2003.

[4] F. Maloberti, Analog Design for CMOS VLSI System. Boston, MA: Kluwer, 2001.

[5] A. Hasting, The Art of Analog Layout. Upper Saddle River, NJ: Prentice-Hall, 2005 


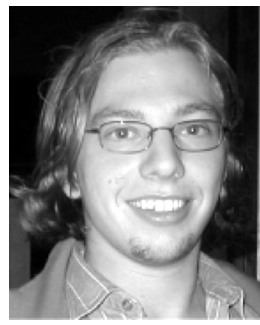

Luca Picolli (S'05) was born in Milan, Italy, in 1980. He received the Laurea degree in electronic engineering from the University of Pavia, Pavia, Italy, in 2005 . He is currently working toward the Ph.D. degree at the Integrated Microsystems Laboratory, University of Pavia.

In the summer of 2006, he held an internship with Conexant to design a $250 \mathrm{MHz} 8$ bit $\mathrm{A} / \mathrm{D}$ converter. His research activities are focused on high-speed and high-resolution A/D converters. He has authored one paper in an international journals and two presentations at international conferences (with published proceedings).

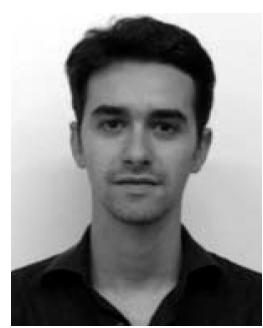

Andrea Rossini (SM'05) was born in Pavia, Italy in 1977. He received the "Laurea" degree in Electronic Engineering from University of Pavia, Italy in 2003. from 2003 to 2006 he joined the Integrated Microsystems Laboratory at the University of Pavia as a Ph.D. student.

His research activities are focused on high speed and high resolution $\mathrm{A} / \mathrm{D}$ converters and interface circuits for integrated sensor. He authored 1 papers in International Journals, and 5 presentations at International Conferences (with published proceedings).

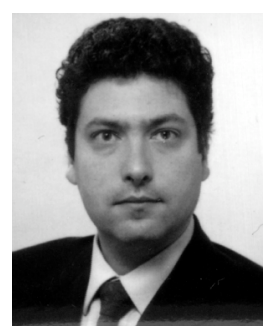

Piero Malcovati (M'95-SM'05) was born in Milan, Italy, in 1968. He received the Laurea degree (summa cum laude) in electronic engineering from the University of Pavia, Pavia, Italy, in 1991, and the Ph.D. degree in electrical engineering from the Swiss Federal Institute of Technology (ETH Zurich), Zurich, Switzerland, in 1996.

From 1996 to 2001, he was an Assistant Professor with the Department of Electrical Engineering, University of Pavia, where he has been an Associate Professor of electrical measurements since 2002. His research activities are focused on microsensor interface circuits and high-performance data converters. He has authored and coauthored more than 30 papers in international journals, more than 100 presentations at international conferences (with published proceedings), five book chapters, and three industrial patents. $\mathrm{He}$ is an Associate Editor of the Journal of Circuits, Systems, and Computers.

Prof. Malcovati was a Guest Editor for the Journal of Analog Integrated Circuits and Signal Processing for the special issue on IEEE ICECS 1999. He served as Special Session Chairman for the IEEE ICECS 2001 Conference, as Secretary of the Technical Program Committee for the ESSCIRC 2002 Conference, and as Technical Program Chairman of the IEEE PRIME 2006 Conference. He was and still is a member of the Scientific Committees for several international conferences, including ESSCIRC, DATE, ECCTD, SENSORS, and PRIME.

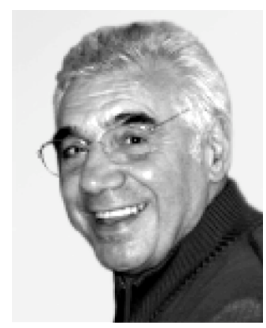

Franco Maloberti (SM'87-F'96) received the Laurea degree in physics (summa cum laude) from the University of Parma, Parma, Italy, in 1968, and the Doctorate Honoris Causa in electronics from the Instituto Nacional de Astrofisica, Opticay Electronica (Inaoe), Puebla, Mexico, in 1996.

$\mathrm{He}$ was a Visiting Professor with the Swiss Federal Institute of Technology (ETH Zurich), Zurich, Switzerland, and with the EPFL, Lausanne, Switzerland. He was the TI/J. Kilby Chair Professor at Texas A\&M University, College Station, and the Distinguished Microelectronic Chair Professor at the University of Texas at Dallas, Richardson. Presently, he is a Professor of Microelectronics and Head of the Micro Integrated Systems Group, University of Pavia, Pavia, Italy. His professional expertise is in the design, analysis, and characterization of integrated circuits and analog digital applications, mainly in the areas of switched-capacitor circuits, data converters, interfaces for telecommunication and sensor systems, and CAD for analog and mixed A/D design. He has authored or coauthored more than 340 published papers and four books and holds 24 patents.
Dr. Maloberti was the recipient of the XII Pedriali Prize for his technical and scientific contributions to national industrial production, in 1992. He was corecipient of the 1996 Institute of Electrical Engineers Fleming Premium. He was the President of the IEEE Sensor Council from 2002 to 2003 and Vice President, Region 8, of the IEEE Circuits and Systems (CAS) Society from 1995 to 1997 and an Associate Editor of the IEEE TRANSACTIONS ON CIRCUITS AND SYSTEMS II. Presently, he is serving as Vice President of Publications of the IEEE CAS Society. He was the recipient of the 1999 IEEE CAS Society Meritorious Service Award, the 2000 CAS Society Golden Jubilee Medal, and the 2000 IEEE Millennium Medal.

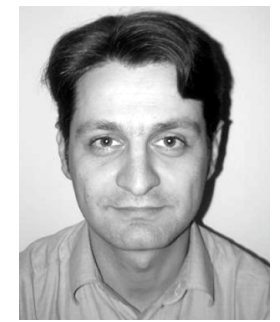

Fausto Borghetti was born in Verona, Italy, in 1974. He received the Laurea and Ph.D. degrees in electronic engineering and computer science from the University of Pavia, Pavia, Italy, in 2000, and 2004, respectively

During that period, his main field of interest was the design of analog-digital converters (ADCs) and circuit interfaces for pixel array sensors devoted to X-ray and gamma-ray spectroscopy. In 2004, he was with Integrated Micro Systems Group (IMS), University of Pavia, working on multiple-output dc-dc buck converters. In 2005, he joined the Integrated Optical Sensors Group, Istituto Trentino di Cultura/Istituto Ricerca Scientifica e Tecnologica (ITC/irst), Povo, Italy, where he is involved in the design of high-performance optical sensors based on APS and SPAD devices. His research interests are in ADC, high-efficiency dc-dc converters, and CMOS image sensors.

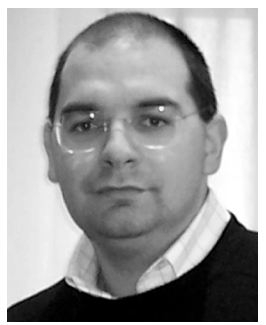

Andrea Baschirotto (M'95-SM'01) received the degree (summa cum laude) and the Ph.D. degree in electronic engineering from the University of Pavia, Pavia, Italy, in 1989 and 1994, respectively, both in electronic engineering.

In 1994, he joined the Department of Electronics, University of Pavia, as a Researcher (Assistant Professor). In 1998, he joined the Department of Innovation Engineering, University of Lecce, Lecce, Italy, as an Associate Professor. Regarding his research activity, he founded and is leading the Microelectronics Group at the University of Lecce, which is collaborating with several companies and research institutions (e.g., IMEC, Infineon, University of Pavia, RFDomus, and STMicroelectronics). His main research interests are in the design of CMOS mixed analog/digital integrated circuits, in particular for low-power and/or high-speed signal processing. He has participated in several research collaborations, also funded by National and European projects. He is/has been responsible of some national and regional projects for the design of ASIC. Since 1989, he has collaborated with several companies on the design of mixed-signal ASICs, including STMicroelectronics, Mikron, ACCO, ITC-IRST, IMEC, and RFDomus (he was a member of their Advisory Board). He has authored or coauthored more than 190 papers in international journals and presentations at international conferences, six book chapters, and holds 25 U.S. patents. In addition, he has coauthored more than 120 papers within research collaborations on high-energy physics experiments. He has long-term experience in microelectronics regarding teaching, researching, and industrial designing. He has been teaching regular academic courses since 1997 . He organized the full educational courses for Electronics Engineering (Bachelor, Master, and Ph.D. programs) at the University of Lecce. He has been leading industrial courses since 1996 (at, for example, Bosch, STMicroelectronics, ITC-IRST, Conexant, and Mikron). He is a speaker at the MEAD Summer courses held at EPFL (Lausanne-Switzerland). He has presented short courses or tutorials at conferences including ISSCC, ISCAS, and PRIME.

Prof. Baschirotto was an Associate Editor for the IEEE TRANSACTIONS ON CIRCUITS AND SYSTEMS II for the period 2000-2003, and for the IEEE TRANSACTIONS ON CIRCUITS AND SYSTEMS I as an Associate Editor for the period 2004-2005. He was the Technical Program Committee Chairman for ESSCIRC 2002 and the Guest Editor for the IEEE JouRnAL OF SOLID-STATE CIRCUITS for ESSCIRC 2003. He was the General Chair of PRIME2006. He is a member of the Technical Program Committee of several international conferences. 